\title{
Effect of Follicle Size on Cumulus-Expansion, In Vitro Fertilization and Development of Porcine Follicular Oocytes
}

\author{
Yun QIAN'), Wei Qun $\mathrm{SHI}^{2)}$, Jia Tong DING ${ }^{3)}$, Bi Qin FAN ${ }^{2)}$ and \\ Yutaka FUKUI ${ }^{4)}$
}

${ }^{1)}$ Research Center of Human Reproduction, The First Affiliated Hospital of Nanjing Medical University, Nanjing 210029, P. R. China, ${ }^{2)}$ Jiangsu Academy of Agricultural Sciences, Nanjing 210014, P. R. China, ${ }^{3}$ College of Animal Science and Veterinary Medicine, Yangzhou University, Yangzhou 225009, P. R. China, ${ }^{4}$ Laboratory of Animal Genetics and Reproduction, Obihiro University of Agriculture and Veterinary Medicine, Obihiro, Hokkaido 080-8555, Japan

\begin{abstract}
The aim of the present experiment was to investigate the effect of follicular size on oocyte cumulus-expansion, in vitro fertilization and subsequent developmental competence. After maturation culture, the rates of porcine oocytes with expanded cumulus derived from antral follicles $\geq 5 \mathrm{~mm}, 4-4.9 \mathrm{~mm}, 3-3.9 \mathrm{~mm}$ and $2-2.9 \mathrm{~mm}$ in diameter were $90.5 \%, 89.7 \%, 85.4 \%$ and $67.4 \%$, respectively. After in vitro fertilization, the developmental competence of oocytes dependently increased with follicular size. Higher cleavage rates and higher proportions of 3-4-cell embryos were obtained from $\geq 4 \mathrm{~mm}$ follicles compared to $2-2.9 \mathrm{~mm}$ follicles $(\mathrm{P}<0.05)$. Although the proportions of 6-8-cell and 12-16-cell embryos obtained from $\geq 5 \mathrm{~mm}, 4-4.9 \mathrm{~mm}, 3-3.9 \mathrm{~mm}$ and 2-2.9 $\mathrm{mm}$ follicles showed no significant differences, embryos obtained from $2-2.9 \mathrm{~mm}$ follicles showed a complete failure to develop beyond the 8-cell stage, embryos obtained from 3-3.9 mm follicles failed to develop beyond the 16-cell stage and none of embryos developed beyond the early stage of morula. The percentages of expanded cumulus-oocyte complexes (COCs) and 2-cell cleavage rates of oocytes derived from 2-2.9 mm follicles were not significantly different when the oocytes were matured for 36 $\mathrm{h}, 42 \mathrm{~h}$ and $48 \mathrm{~h}$ in vitro. The exposure to $5 \%$ or $15 \%$ porcine follicular fluid (PFF) from the large follicles during in vitro maturation (IVM) had no significant effect on the cleavage and subsequent developmental rates of porcine oocytes compared to PFF from small follicles.
\end{abstract}

Key words: Porcine oocytes, Cumulus-expansion, In vitro fertilization, In vitro development, Follicular size

(J. Reprod. Dev. 47: 145-152, 2001)

I $\mathrm{n}$ mammals, meiotic division of the oocyte is initiated during fetal life and is arrested at the diplotene stage of prophase I shortly before or after the birth. The oocyte remains under meiotic arrest until the ovulatory luteinizing hormone $(\mathrm{LH})$ surge, which stimulates the resumption of meiosis in the Graafian follicle. It is also known that

Accepted for publication: March 2, 2001

Correspondence: Y. Fukui mammalian follicular oocytes undergo spontaneous meiotic maturation when liberated from follicles and cultured in an appropriate medium [1].

Recently, it has been described that the oocyte from the dominant follicle underwent further ultrastructural modifications and attained full developmental competence through a process that might be termed "capacitation" [2]. Ding et al. [3] 
reported that follicular maturation is paralleled by, and functionally related to, the maturation of the oocyte inside the follicle. The results confirmed that diversity of follicular development may affect the oocyte quality and that oocytes would be at different developmental stages when derived from different sized follicles. The developmental competence of follicular oocytes in vitro may be affected by the follicular sizes [4]. Some researchers investigated the effect of follicular size on oocyte quality and developmental competence following in vitro maturation (IVM), fertilization (IVF), and culture (IVC) in bovine and goat [5-8]. It is well recognized that the characteristics of follicles could be an important parameter to determine the developmental competence of oocytes fertilized in vitro.

Porcine oocytes from antral follicles $(2-6 \mathrm{~mm}$ in diameter) on the surface of slaughtered ovaries have been collected for in vitro production of porcine embryos [9-11]. However, there is a large variation in reported IVM-IVF results among laboratories [12]. Prather and Day [12] reported that even though only cumulus-oocyte complexes (COCs) with uniform ooplasm and compact cumulus cell mass were usually selected for oocyte maturation, there was a large variation in the dictyate stage of the first meiotic prophase among oocytes collected from follicles of different sizes. Therefore, the quality of oocytes collected from different follicular sizes, or from different ages might be a major source of variation in IVM results. Furthermore, following IVM and IVF only 3\% of the embryos developed into blastocysts after in vitro culture $[11,13]$. From these data, it is clear that only a small proportion of porcine oocytes selected for IVM can complete full cytoplasmic maturation, which confers the ability to support embryonic development. So it is necessary to further investigate the effects of differences in the follicular environment on oocyte quality.

The objectives of the present study were 1) to investigate the effect of follicular size on the type of porcine oocytes obtained for IVM and on the ability of such oocytes to be fertilized and undergo cleavage and early embryonic development in vitro; and 2) to assess the effect of using different proportions of porcine follicular fluid (PFF) from follicles of different sizes in the IVM medium on cumulus-expansion and in vitro development (IVD) of porcine oocytes.

\section{Materials and Methods}

\section{Collection of follicular oocytes}

Ovaries were obtained from perpubertal gilts at a local slaughterhouse and transported to the laboratory within $2 \mathrm{~h}$ in saline $(9 \mathrm{~g} \mathrm{NaCl} / 1,100 \mathrm{IU}$ penicillin $\mathrm{GK} / \mathrm{ml}, 100 \mathrm{IU}$ streptomycin sulphate/ $\mathrm{ml}$ ) at 37-38 C. Ovaries were immediately freed from their hilus and connective tissues, and washed 2-3 times in saline at $37 \mathrm{C}$. COCs were aspirated with a $10-\mathrm{ml}$ syringe equipped with a 19 -gauge needle. Only oocytes with compact cumulus cells were selected. They were washed 3 times in Dulbecco's-PBS (D-PBS) with 5\% newborn calf serum (NCS: Gibco; Cat. No. 16010-159) (pH: 7.48) for the experiments.

\section{In-vitro maturation (IVM)}

COCs were washed 3 times with IVM medium, which consisted of TCM199 (Gibco; Cat. No. 31100035), 10\% fetal calf serum (FCS: Gibco; Cat. No. 16000-036), 10 IU/ml eCG (Ninbo, China; Cat. No. 981018), $10 \mathrm{IU} / \mathrm{ml}$ hCG (Ninbo, China; Cat. No. 980630), $1 \mu \mathrm{g} / \mathrm{ml}$ estradiol $-17 \beta$ (Fluka, Switzerland; EEC No. 2000238), $100 \mathrm{mg} / 1$ sodium pyruvate, $900 \mathrm{mg} / 1$ calcium lactate, $550 \mathrm{mg} / \mathrm{l} \mathrm{D}$ glucose, $5958 \mathrm{mg} / 1$ Hepes, and $100 \mathrm{IU} / \mathrm{ml}$ penicillin GK, $100 \mathrm{IU} / \mathrm{ml}$ streptomycin sulphate ( $\mathrm{pH}: 7.38$ ), then transferred to a droplet of IVM medium (10-15 oocytes /100 $\mu \mathrm{l}$ ) under mineral oil (Sigma; Lot. 103H10455) in a polystyrene dish (35 mm: Nunclon, Denmark; Cat. No. 153066), and cultured for $36 \mathrm{~h}$ at $39 \mathrm{C}$ under $5 \% \mathrm{CO}_{2}$ in a $\mathrm{CO}_{2}$ incubator (Asahi, Japan; Model 4020). After culture, oocytes with expanded cumulus mass were selected and subjected to insemination.

\section{Sperm preparation}

The sperm preparation was carried out using a modification of the method described by Nagai et al. [14]. The epididymides of the boar (Landrace $\times$ Meishan $\times$ Yorkshire, 8 months old) were removed at the slaughterhouse and returned to the laboratory in saline at $37 \mathrm{C}$ within $15 \mathrm{~min}$. The epididymal spermatozoa were extruded from the distal portion of the cauda by pressure with a $20-\mathrm{ml}$ syringe, then diluted with BF-3 solution (1:3) (BF-3 solution: $40 \mathrm{~g} / 1$ lactose, $20 \mathrm{~g} / 1$ casein, $20 \mathrm{~g} / 1$ Tris, 10 $\mathrm{g} / 1$ citric acid monohydrate, $10 \mathrm{~g} / 1$ sucrose) and kept for $2 \mathrm{~h}$ at $4 \mathrm{C}$. Next the solution was diluted 
with $4 \%$ glycerol (1:1) and balanced for $2 \mathrm{~h}$ at $4 \mathrm{C}$. After balance, the sperm suspensions were frozen in $0.1 \mathrm{ml}$ pellets and stored in liquid nitrogen until required for IVF.

Frozen spermatozoa (3 pellets) were thawed in 10 $\mathrm{ml}$ D-PBS containing 5\% NCS, $2 \mathrm{mg} / \mathrm{ml}$ BSA-V (Böchringer, Germany) (pH: 7.48) at $37 \mathrm{C}$. Immediately after thawing, the suspensions were centrifuged for $4 \mathrm{~min}$ at $200 \mathrm{~g}$, the supernatant was discarded and the spermatozoa were subsequently diluted to $2-4 \times 10^{8}$ cells $/ \mathrm{ml}$ and preincubated in sperm preincubation medium which consisted of TCM199 with Earle's salts, 12\% FCS , $0.9 \mathrm{mg} / 1$ calcium lactate, $0.1 \mathrm{~g} / 1$ sodium pyruvate, $0.55 \mathrm{~g} / 1$ D-glucose, $5.958 \mathrm{~g} / 1$ Hepes, $100 \mathrm{IU} / \mathrm{ml}$ penicillin GK, and $100 \mathrm{IU} / \mathrm{ml}$ streptomycin sulfate $(\mathrm{pH}: 7.80)$, at $37 \mathrm{C}$ under $5 \% \mathrm{CO}_{2}$ in air for $1 \mathrm{~h}$. After preincubation, the proportion of spermatozoa with progressive forward motility was more than $60 \%$.

\section{In-vitro fertilization(IVF), examination of oocytes and embryos culture}

The oocytes with expanded cumulus mass were washed three times and transferred to the fertilization medium, BO solution containing 10 $\mathrm{mg} / \mathrm{ml} \mathrm{BSA}-\mathrm{V}$, and $2 \mathrm{mM}$ caffeine ( $\mathrm{pH}:$ 7.48). A portion of the preincubated sperm suspension was introduced into the medium so that the final concentration for IVF was $2-4 \times 10^{7}$ cells $/ \mathrm{ml}$.

After incubation for $12-16 \mathrm{~h}$, the oocytes were removed from the surrounding cumulus mass and spermatozoa by agitation using a narrow-bore glass pipette and washed twice with an embryo culture medium which consisted of TCM199 with Earle's salts, $10 \%$ FCS, $3.7 \mathrm{ml} / 1$ sodium lactate $(60 \%$ syrup), $40 \mathrm{mg} / 1$ sodium pyruvate, $5958 \mathrm{mg} / 1$ Hepes, $100 \mathrm{IU} / \mathrm{ml}$ penicillin GK, and $100 \mathrm{IU} / \mathrm{ml}$ streptomycin sulfate $(\mathrm{pH}: 7.40)$. Then 10-15 oocytes were transferred to a droplet of the embryo culture medium $(100 \mu \mathrm{l})$ containing granulosa cells prepared by the method described below.

To determine the rate of fertilization, some embryos were randomly selected after insemination and fixed for 3-4 days with acetic alcohol (methanol and acetic acid, 3:1, v/v) at $4 \mathrm{C}$, stained with $1 \%$ aceto-orcein and examined under a phase-contrast microscope (Olympus, Japan). Oocytes having enlarged sperm heads and/or male pronuclei with sperm tails were regarded as fertilized. Among the fertilized oocytes, fertilization was considered normal fertilization if a sperm-tail closed to a male pronucleus in the cytoplasm observed.

\section{Preparation of granulosa cells}

The oocytes with expanded cumulus mass were treated with $0.1 \%$ hyaluronidase (Sigma; H6254), and the suspended granulosa cells were washed 23 times with the embryo culture medium. Then, the cells were cultured at a concentration of $1 \times 10^{5}$ cells $/ \mathrm{ml}$ in the same medium until confluency and used for the co-cultivation with the embryos.

\section{Experimental design and statistical analysis}

In Experiment 1, the effects of follicular size on porcine oocytes obtained for IVM and on the ability of such oocytes to develop in vitro following IVM/ IVF/IVC were examined. On recovery, oocytes were classified into four groups according to the size of the follicles ( $\mathrm{mm}$ in diameter) from which the oocytes were derived (group 1: 2-2.9 mm; group 2: 3-3.9 mm; group 3: 4-4.9 mm; group 4: $\geq 5$ $\mathrm{mm})$.

In Experiment 2, the effects of different culture times ( $36 \mathrm{~h}, 42 \mathrm{~h}$ and $48 \mathrm{~h}$ ) during in vitro maturation on cumulus-expansion and the developmental competence of oocytes derived from 2-2.9 mm follicles were tested.

In Experiment 3, the effects of different concentrations (5\% and $15 \%$ ) of porcine follicular fluid (PFF) from follicles of different sizes (group 1: 2-2.9 mm; group 2: 3-3.9 mm; group 3: 4-4.9 mm; group 4: $\geq 5 \mathrm{~mm}$ ) during IVM on cumulusexpansion and the developmental competence of porcine oocytes from $>2 \mathrm{~mm}$ follicles were investigated. Each PFF sample aspirated from different sized follicles was centrifuged at $1,000 \mathrm{~g}$ for 20 minutes at room temperature to remove blood cells and debris. The supernatant was transferred to a sterile centrifuge tube and stored at $-20 \mathrm{C}$ until use. PFF was passed through $0.22-\mu \mathrm{m}$ membrane filters before use.

Data were analyzed with ANOVA, $\chi^{2}$-test and differences were considered to be significant at $\mathrm{P}<0.05$.

\section{Results}

\section{Experiment 1}

The results of Experiment 1 are presented in Tables 1 and 2. After COCs were cultured for $36 \mathrm{~h}$, 
Table 1. Effect of follicular size on cumulus-expansion of porcine oocytes

\begin{tabular}{ccc}
\hline Group & $\begin{array}{c}\text { No.of oocytes } \\
\text { cultured }\end{array}$ & $\begin{array}{c}\text { No.of oocytes } \\
\text { with expanded cumulus }(\%)\end{array}$ \\
\hline 1 & 141 & $95(67.4)^{\mathrm{a}}$ \\
2 & 164 & $140(85.4)^{\mathrm{b}}$ \\
3 & 78 & $70(89.7)^{\mathrm{b}}$ \\
4 & 63 & $57(90.5)^{\mathrm{b}}$ \\
\hline
\end{tabular}

a, b Values in the same column with different superscripts differ significantly $(\mathrm{p}<0.05)$.

Table 2. Effect of follicular size on in vitro development of porcine oocytes after IVF

\begin{tabular}{cccccccc}
\hline \multirow{2}{*}{ roup } & No. of oocytes & \multicolumn{5}{c}{ In vitro development to (\%) } \\
\cline { 2 - 7 } & used for IVF & 2-cell & 3-4-cell & 6-8-cell & 12-16-cell & Early morula & Beyond morula \\
\hline 1 & 92 & $13(14.1)^{\mathrm{a}}$ & $2(15.4)^{\mathrm{d}}$ & $1(7.7)$ & 0 & 0 & 0 \\
2 & 134 & $28(20.9)^{\mathrm{ab}}$ & $11(39.3)^{\mathrm{de}}$ & $6(21.4)$ & $1(3.6)$ & 0 & 0 \\
3 & 63 & $21(33.3)^{\mathrm{cb}}$ & $11(52.4)^{\mathrm{e}}$ & $6(28.6)$ & $2(9.5)$ & $1(4.8)$ & 0 \\
4 & 54 & $26(48.1)^{\mathrm{c}}$ & $17(65.4)^{\mathrm{e}}$ & $9(34.6)$ & $2(7.7)$ & $1(3.8)$ & 0 \\
\hline
\end{tabular}

$a, b, c, d, e$ Values in the same column with different superscripts differ significantly $(p<0.05)$.

the COCs with expanded cumulus mass were selected for IVF. The rates of oocytes with expanded cumulus from the four different groups were $67.4 \%, 85.4 \%, 89.7 \%$, and $90.5 \%$, respectively. A significant difference between groups 1 vs. 2, 3 and 4 was calculated $(\mathrm{P}<0.05)$, while the difference among groups 2, 3 and 4 was not significant (Table 1).

To examine the fertilization rate, oocytes after incubation for 12-16 h with spermatozoa were randomly selected for fixing and staining. The percentage of oocytes penetrated was $75.6 \%$ (34/ $45)$, the rate of polyspermy was $23.5 \%(8 / 34)$ and the normal fertilization rate was $61.8 \%(21 / 34)$.

The oocytes used for IVF were co-cultured with granulosa cell monolayers. The cleavage rates and the proportions of 3-4-cell, 6-8-cell embryos increased progressively with follicular sizes. The cleavage rates and the proportions of 3-4-cell embryos in groups 3 and 4 were significantly higher $(\mathrm{P}<0.05)$ than those in group 1. Although the proportions of 6-8-cell and 12-16-cell embryos showed no significant differences among groups, embryos in group 1 did not develop beyond the 8cell stage. Also, embryos in group 2 failed to develop beyond the 16-cell stage and all embryos did not develop beyond the early stage of morula (Table 2).

\section{Experiment 2}

The rates of COCs with expanded cumulus derived from $2-2.9 \mathrm{~mm}$ follicles were $67.4 \%, 61.9 \%$ and $71.7 \%$ for $36 \mathrm{~h}, 42 \mathrm{~h}$ and $48 \mathrm{~h}$ culture, respectively, and the cleavage rates of 2-cell embryos were $14.1 \%, 17.4 \%$ and $11.4 \%$ without significant differences among them (Table 3 ).

\section{Experiment 3}

The results of Experiment 3 are presented in Tables 4 and 5. Neither the rates of COCs with expanded cumulus nor the cleavage rates differed significantly between treatments. With regard to the cleavage rate, supplementation of $5 \%$ and $15 \%$ PFF from large follicles into the IVM medium resulted in higher cleavage rate than those cultured in the medium supplemented with PFF from small follicles, but there was no significant difference between treatments.

\section{Discussion}

It is well recognized that oocytes recovered from slaughterhouse materials for in vitro embryo production are extremely heterogeneous in terms of quality and developmental competence [15]. The widespread use of in vitro-produced porcine embryos has emphasized the necessity for non- 
Table 3. Effect of culture time on cumulus-expansion and IVD of porcine oocytes derived from 2-2.9 mm follicles

\begin{tabular}{cccccccc}
\hline $\begin{array}{c}\text { Culture time } \\
(\mathrm{h})\end{array}$ & $\begin{array}{c}\text { No. of oocytes } \\
\text { cultured }\end{array}$ & \multicolumn{2}{c}{$\begin{array}{c}\text { No. of oocytes with } \\
\text { expanded cumulus(\%) }\end{array}$} & $\begin{array}{c}\text { No. of oocytes } \\
\text { used for IVF }\end{array}$ & \multicolumn{4}{c}{ In-vitro development to (\%) } \\
\cline { 6 - 8 } & & $95(67.4)$ & 92 & $13(14.1)$ & $2(15.4)$ & $1(7.7)$ & 0 \\
46 & 141 & $26(61.9)$ & 23 & $4(17.4)$ & 0 & 0 & 0 \\
42 & 42 & $38(71.7)$ & 35 & $4(11.4)$ & $1(25.0)$ & 0 & 0 \\
\hline 8
\end{tabular}

Table 4. Effect of different concentrations of porcine follicular fluid (PFF) from different follicle sizes during IVM on cumulus-expansion of porcine oocytes

\begin{tabular}{crcc}
\hline PFF from group & PFF conc. & $\begin{array}{c}\text { No. of oocytes } \\
\text { cultured }\end{array}$ & $\begin{array}{c}\text { No. of oocytes with } \\
\text { expanded cumulus (\%) }\end{array}$ \\
\hline 1 & $15 \%$ & 81 & $67(82.7)$ \\
2 & $5 \%$ & 85 & $69(81.2)$ \\
& $15 \%$ & 91 & $75(82.4)$ \\
3 & $5 \%$ & 91 & $73(80.2)$ \\
& $15 \%$ & 70 & $62(88.6)$ \\
4 & $5 \%$ & 90 & $74(82.2)$ \\
& $15 \%$ & 68 & $75(83.7)$ \\
& $5 \%$ & 90 & $74(81.3)$ \\
\hline
\end{tabular}

Table 5. Effect of different concentrations of porcine follicular fluid (PFF) from different follicle sizes during IVM on IVD of oocytes

\begin{tabular}{|c|c|c|c|c|c|c|c|c|}
\hline \multirow{2}{*}{$\begin{array}{l}\text { PFF from } \\
\text { group }\end{array}$} & \multirow[t]{2}{*}{ PFF conc. } & \multirow{2}{*}{$\begin{array}{l}\text { No. of oocytes } \\
\text { used for IVF }\end{array}$} & \multicolumn{6}{|c|}{ In vitro development to (\%) } \\
\hline & & & 2-cell & 3-4-cell & 6-8-cell & 12-16-cell & Early morula & Beyond morula \\
\hline \multirow[t]{2}{*}{1} & $15 \%$ & 67 & $30(44.8)$ & $11(36.7)$ & $4(13.3)$ & $1(3.3)$ & 0 & 0 \\
\hline & $5 \%$ & 69 & $25(36.2)$ & $6(24.0)$ & $2(8.0)$ & 0 & 0 & 0 \\
\hline \multirow[t]{2}{*}{2} & $15 \%$ & 75 & $33(44.0)$ & $12(36.4)$ & $6(18.2)$ & $1(3.0)$ & 0 & 0 \\
\hline & $5 \%$ & 73 & $30(41.1)$ & $9(30.0)$ & $4(13.3)$ & 0 & 0 & 0 \\
\hline \multirow[t]{2}{*}{3} & $15 \%$ & 62 & $26(41.9)$ & $11(42.3)$ & $7(26.9)$ & $2(7.7)$ & 0 & 0 \\
\hline & $5 \%$ & 74 & $30(40.5)$ & $12(40.0)$ & $6(20.0)$ & $2(6.6)$ & 0 & 0 \\
\hline \multirow[t]{3}{*}{4} & $15 \%$ & 61 & $29(47.5)$ & $13(44.8)$ & $8(27.6)$ & $3(10.3)$ & $2(10.3)$ & 0 \\
\hline & $5 \%$ & 75 & $34(45.3)$ & $14(41.2)$ & $9(26.5)$ & $3(8.8)$ & $1(2.9)$ & 0 \\
\hline & $0 \%$ & 73 & $26(35.6)$ & $12(46.2)$ & 7 (26.9) & $2(7.7)$ & $1(3.8)$ & 0 \\
\hline
\end{tabular}

invasive methods for the selection of oocytes competent for in vitro development. Such selection may be exerted at various points along the developmental axis and thus may be based on one or more of several parameters, including the characteristics of the enclosing follicle.

Procházka et al. [16] demonstrated that IVM rate and cumulus-expansion of porcine COCs were affected by the sizes of the donor follicles, but they did not further investigate the relationship between the follicular size and the developmental competence of oocytes fertilized in vitro. To our knowledge, the present study is the first to demonstrate a clear relationship between follicular size and cumulus-expansion, IVF and IVD in porcine oocytes.

As reported in porcine, meiotic competence is acquired progressively during follicular growth [17]. Full maturation involves both nuclear and cytoplasmic events that confer on the oocyte capacity for supporting normal fertilization and early embryonic development [12]. In agreement with the findings from goat and bovine, with increase of the follicular size, the developmental competence of porcine oocytes fertilized in vitro increased gradually in the present study. As 
pointed out by Sirard et al. [18], oocytes accumulate a very stable form of RNA that is translated during maturation, fertilization, and early embryonic development. The RNA accumulation could be well influenced by the quality of the follicular microenvironment. Fluctuations in this microenvironment may result in variable developmental potential following fertilization [19]. Tan and Lu [20] and McCaffrey et al. [21] concluded that a factor inherent in oocytes derived from smaller follicles limited their further development in bovine. Barnes et al. also observed that follicle size can greatly affect oocyte developmental competence and concluded that the factors required for improved development are to be found within the cytoplasm of the oocytes derived from the larger follicles [5]. It is well known that nuclear events within oocytes derived from $>2 \mathrm{~mm}$ follicles are at a minimum. The transcripts produced during this period of low transcriptional activity are critical for development and the improved developmental competence is due to cytoplasmic maturation of the oocytes during the final phases of folliculogenesis. Such follicles, although not preovulatory, are presumably among those competing for dominance and thus may provide an environment more conducive to proper cytoplasmic maturation, giving their oocytes greater capacity for embryonic development. The results reported here suggest that the competence to undergo cytoplasmic maturation is also acquired progressively by porcine oocytes during follicular growth. Therefore possible explanations for the relationship between follicular size and oocyte quality may be: 1) a factor inherent in the oocytes derived from the smaller follicles limits their further development; 2) the cytoplasm of oocytes derived from the larger follicles may have some factors required for improved development; and 3) the larger follicles may provide a better environment to induce cytoplasmic maturation for supporting subsequent embryonic development.

In an effort to determine if some special ingredient was contained in the follicular environment of the oocytes from large follicles, PFF from the different sized follicles was used in the IVM medium. The results indicate that supplementation of PFF from large follicles into the IVM medium is likely favorable to the acquisition of developmental competence by immature oocytes.

For some reason, culture time for maturation may affect IVM, IVF and IVD of oocytes. Especially, oocytes derived from the smaller follicles may require a longer culture time for maturation in vitro. In order to examine the effects of culture time on IVM and IVD of COCs, oocytes derived from 2-2.9 $\mathrm{mm}$ follicles were cultured in vitro for $36 \mathrm{~h}, 42 \mathrm{~h}$ and $48 \mathrm{~h}$. The results indicated that cumulus-expansion and the development competence of oocytes in the three groups were not significantly different, suggesting that before being isolated from follicles, the oocytes had already grown beyond some point for synthesizing some specific RNAs and proteins, then acquired the full competence to support the development of oocytes fertilized in vitro. Those changes and accumulation in the oocytes did not occur in vitro, even though the culture time in vitro was prolonged, thus the developmental competence may be related to the different developmental stages of oocytes before culture in vitro [22].

The embryo culture medium used in the present study was the same medium reported by Yoshida et al. [11]. Yoshida et al. [11] demonstrated that this culture medium may not have been suitable for embryos beyond the 2-cell stage due to lactate and pyruvate added to the medium. The detrimental effect of lactate and pyruvate on pig embryo development has been reported by Davis and Day [23] and Davis [24]. The present results showed that some embryos could develop beyond the 4-cell stage when co-cultured with granulosa cell monolayers. To some extent, the presence of somatic cells can overcome the detrimental effects of lactate and pyruvate on pig embryo development in vitro. Our study supports the hypothesis that the co-culture system could remove some toxic compounds from the culture medium. Generally, most of porcine embryos developing beyond the 4-cell stage can develop to the blastocyst stage. However, in the present study, "Developmental Block" seemed to exist at each cleavage stage and more than $10 \%$ of embryos lost their viability at each cleavage stage when cultured in vitro. Possible explanations for this are: 1) the viability of embryos generated in vitro, and 2) the culture conditions following in vitro fertilization are not appropriate for porcine zygotes. It is well known that different metabolic requirements are required at the different developmental stages of 
embryos during in vitro culture [25, 26].

In summary, the following conclusions can be drawn from the present study: 1) there is a clear relationship between follicular size and porcine oocyte quality in terms of cumulus-expansion, IVF and IVD; 2) the developmental competence is acquired progressively by the porcine oocyte during follicular growth. 3) the developmental competence of oocytes derived from 2-2.9 mm follicles cultured for $36 \mathrm{~h}, 42 \mathrm{~h}$ and $48 \mathrm{~h}$ was not significantly different. 4) supplementation of the IVM medium with PFF from large follicles is to some extent favorable to the acquisition of developmental competence by immature oocytes; and 5) porcine embryos can overcome
"Developmental Block" when co-cultured with granulosa cell monolayers, and different culture conditions based on the different stages of porcine embrio development are required to optimize an in vitro culture system.

\section{Acknowledgements}

The authors thank to Prof. S. Zhong for supply of the porcine epididymal spermatozoa, Dr. HL. Liu and Dr. HH. Wang for technical assistance. This study was part of the Chinese-Japan co-operation program in Agricultural Science.

\section{References}

1. Pincus G, Enzmann EV. The comparative behavior of mammalian eggs in vivo and in vitro.1. The activation of ovarian eggs. J Exp Med 1935; 62: 665675.

2. Hyttel P, Fair T, Callesen H, Greve T. Oocyte growth, Capacitation and final maturation in cattle. Theriogenology 1997; 47: 23-32.

3. Ding J, Foxcroft GR. Conditioned media produced by follicular shells of different maturity affect maturation of pig oocytes. Biol Reprod 1994; 50: 1377-1384.

4. Ding J, Foxcroft GR. Follicular heterogenecity and oocyte maturation in vitro in pig. Biol Reprod 1992; 47: 648-655.

5. Lonergan P, Monaghan P, Boland MP, Gordon I. Effect of follicle size on bovine oocyte quality and developmental competence following maturation, fertilization, and culture in vitro. Mol Reprod Dev 1994; 37: 48-53.

6. Fair T, Hyttel P, Greve T. Bovine oocyte diameter in relation to maturational competence and transcriptional activity. Mol Reprod Dev 1996; 42: 437-442.

7. De smedt V, Grozet N, Gall L. Morphological and functional changes accompanying the acquisition of meiotic competence in ovarian goat oocyte. J Exp Zool 1994; 269: 128-139.

8. Crozet N, Ahmed-Ali M, Dubos MP. Developmental competence of goat oocytes, from follicles of different size categories following maturation, fertilization and culture in vitro. $J$ Reprod Fertil 1995; 103: 293-298.

9. Ding J, Moor RM, Foxcroft GR. Effect of protein synthesis on maturation, sperm penetration, and pronulcear development in porcine oocytes. Mol Reprod Dev 1992; 33: 59-66.
10. Rath $\mathbf{D}$, Niemann $\mathbf{H}$, Tao $\mathbf{T}$. In vitro maturation of porcine oocytes in follicular fluid with subsequent effects on fertilization and embryo yield in vitro. Theriogenology 1995; 44: 529-538.

11. Yoshida M, Ishizaki Y, Kawagishi H. Blastocyst formation by pig embryos resulting from in vitro fertilization of oocytes matured in vitro. $J$ Reprod Fertil 1990; 88: 1-8.

12. Prather RS, Day BN. Practical considerations for the in vitro production of pig embryos. Theriogenology 1998; 49: 23-32.

13. Funahashi H, Stumpf TT, Terlouw SL. Developmental ability of porcine oocytes matured and fertilized in vitro. Theriogenology 1994; 41: 14251433.

14. Nagai T, Takahashi T, Masuda H. In-vitro fertilization of pig oocytes by frozen boar spermatozoa. J Reprod Fertil 1988; 84: 585-591.

15. Gordon I, Lu KH. Production of embryos in vitro and its impact on livestock production. Theriogenology 1990; 33: 77-87.

16. Proch_zka R, Kal_b P, Miyano T. EGF stimulated expansion of porcine oocyte-cumulus complexes is affected by the size of the donor follicle. Theriogenology 1997; 1: 199 (abstract).

17. Motlik J, Crozet N, Fulka J. Meiotic competence in vitro of pig oocytes isolated from early antral follicles. J Reprod Fertil 1984; 72: 323-328.

18. Sirard MA, Coenan K, Bilodeau S. Effects of fresh or cultured follicular fractions on meiotic resumption in bovine oocytes. Theriogenology 1992; 37: 39-57.

19. Moor RM, Powell DJ. Translational capacity of sheep oocytes microinjected with messanger RNA. J Reprod Fertil 1989; 86: 289-295.

20. Tan SJ, Lu KH. Effects of different oestrous cycle 
stages of ovaries and sizes of follicles on generation of IVF early embryos. Theriogenology 1990; 33: 335 (abstract).

21. McCaffrey C, Lu KH, Seenan JM. Factors involved in the in vitro development of IVF cattle ova. In: Proceeding of the Irish Grassland and Animal Production Association, 18th Annual Research meeting. Dubbin: O'kiely 1992; 33-34.

22. Eppig JL, Schroeder AC, O'Brien MJ. Developmental capacity of mouse oocyte matured in vitro: effects of gonadotrophic stimulation, follicular origin and oocyte size. J Reprod Fertil 1992; 95: 119-127.

23. Davis DL, Day BN. Cleavage and blastocyst formation by pig embryos in vitro. J Anim Sci 1978; 46: 1043-1053.

24. Davis DL. Culture and storage of pig embryos. J Reprod Fertil 1985; 33 (Suppl): 115-124.

25. Lee ES, Fukui Y. Synergistic effect of alanine and glycine on bovine embryos cultured in a chemically defined medium and amino acid uptake by in vitroproduced bovine morulae and blastocysts. Biol Reprod 1996; 55: 1383-1389.

26. Fukui Y, Lee ES, Araki N. Effect of medium renewal during culture in two different culture systems on development to blastocysts from in vitro produced early bovine embryos. J Anim Sci 1996; 74: 2752-2758. 